\title{
TEST OF CPT AND LORENTZ INVARIANCE FROM MUONIUM SPECTROSCOPY
}

\author{
D. KAWALL, V. W. HUGHES, M. GROSSE PERDEKAMP ${ }^{a}$, W. LIU \\ Department of Physics, Yale University, \\ New Haven, CT 06520-8120, USA \\ E-mail: david.kawall@yale.edu \\ K. JUNGMANN ${ }^{b}$, G. ZU PUTLITZ \\ Physikalisches Institut, Universität Heidelberg, \\ D-69120 Heidelberg, Germany
}

\begin{abstract}
Following a suggestion of Kostelecký et al. we have evaluated a test of CPT and Lorentz invariance from the microwave spectroscopy of muonium. Violations of CPT and Lorentz invariance in the muon sector would be indicated by frequency shifts $\delta \nu_{12}$ and $\delta \nu_{34}$ in $\nu_{12}$ and $\nu_{34}$, the two hyperfine transitions involving muon spin flip, which were precisely measured in ground state muonium in a strong magnetic field of $1.7 \mathrm{~T}$. Such shifts would appear in the laboratory frame as anticorrelated oscillations in $\nu_{12}$ and $\nu_{34}$ at the earth's sidereal frequency. Our experiment found no time dependence in $\nu_{12}$ or $\nu_{34}$ at the level of $20 \mathrm{~Hz}$, limiting the size of some CPT and Lorentz violating parameters at the level of $2 \times 10^{-23}$ $\mathrm{GeV}$, representing for the moment the most sensitive limits on some of the muon parameters of the theory.
\end{abstract}

\section{Introduction}

Muonium $\left(\mu^{+} e^{-}, M\right)$ is the hydrogenlike bound state of a positive muon and electron. Interest in this simple atom arises for several reasons. For precision tests of QED, muonium is well suited since for instance, unlike for hydrogen, its ground state hyperfine structure can be calculated with high precision since the complications of hadronic structure are absent. The combination of precise calculations and experiments enable precision tests of QED to be performed, and also allows the accurate determination of fundamental constants 1.2. Muonium is also used in searches for physics beyond the standard model, specifically lepton number violation 6 , since it combines leptons from two different generations.

Another test of fundamental physics became possible after the development by Kostelecký and coworkers 16 of a plausible mechanism by which CPT and Lorentz symmetry might be violated, based on spontaneous breaking of $\mathrm{CPT}$ and Lorentz symmetry in an underlying higher order theory. The new

${ }^{a}$ Current Address : Riken-BNL Research Center, Upton, NY 11973, USA

${ }^{b}$ Current Address : KVI, Zernikelaan 25, NI-9747 AA Groningen, The Netherlands 


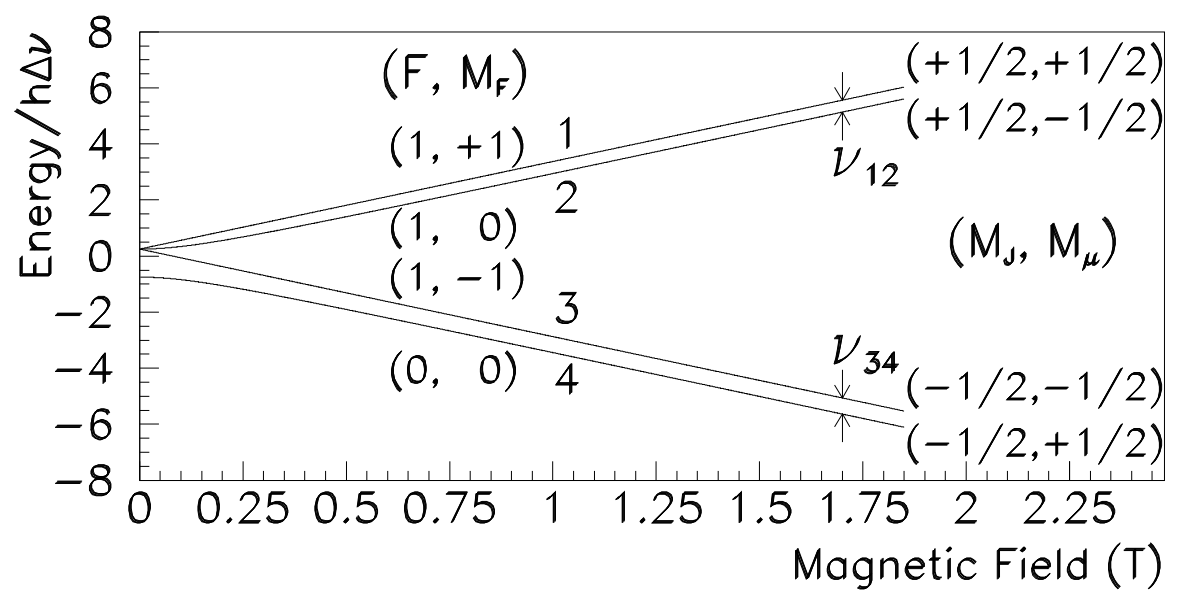

Figure 1: Breit-Rabi energy level diagram of ground state muonium. At high fields, the indicated transitions, $\nu_{12}$ and $\nu_{34}$ are essentially muon spin flip transitions.

phenomenology resulting from $\mathrm{CPT}$ and Lorentz violation was incorporated in extensions to the standard model, and its implications for muonium, which prompted this work (more details of which may be found in 1 ), were described in 9 and R. Bluhm's talk at this conference.

The QED extension of the theory leads to potentially observable perturbations in the $1^{2} S_{1 / 2}$ ground state energy levels of muonium, where in a strong field, the ground state splits into four substates labelled 1 through 4 in order of decreasing energy, defined by the magnetic quantum numbers $\left(M_{J}, M_{\mu}\right)$ (see Fig.11). Of the six possible ground state hyperfine transitions we focus on $(1 / 2,1 / 2) \leftrightarrow(1 / 2,-1 / 2)$ designated $\nu_{12}$, and $(-1 / 2,-1 / 2) \leftrightarrow(-1 / 2,1 / 2)$ designated $\nu_{34}$, which were are observed by a microwave magnetic resonance techniquel. The transition frequencies are given by the Breit-Rabi formula

$$
\begin{aligned}
& \nu_{12}=-\frac{\mu_{B}^{\mu} g_{\mu}^{\prime} H}{h}+\frac{\Delta \nu}{2}\left[(1+x)-\sqrt{1+x^{2}}\right], \\
& \nu_{34}=+\frac{\mu_{B}^{\mu} g_{\mu}^{\prime} H}{h}+\frac{\Delta \nu}{2}\left[(1-x)+\sqrt{1+x^{2}}\right],
\end{aligned}
$$

where $x=\left(g_{J} \mu_{B}^{e}+g_{\mu}^{\prime} \mu_{B}^{\mu}\right) H /(h \Delta \nu)$ is a dimensionless parameter proportional to the magnetic field strength, $H$, and $\Delta \nu$ is the ground state hyperfine interval. We used the Larmor relation, $2 \mu_{p} H=h \nu_{p}$, and NMR to determine $H$ in terms of the free proton precession frequency, $\nu_{p}$, and the proton magnetic moment, 
$\mu_{p}$. The electron and muon $g$ factors in muonium, $g_{J}$ and $g_{\mu}^{\prime}$ respectively, differ from their free space by binding corrections of order $\alpha^{2}$ (seet). The experiment, performed at the Clinton P. Anderson Meson Physics Facility at Los Alamos, measured the ground state hyperfine interval $\Delta \nu=\nu_{12}+\nu_{34}$ to 12 ppb precision to compare with theory as a precise test of QED, and also, using (11), (2) and the measurements of the transition frequencies $\nu_{12}$ and $\nu_{34}$ (each made to about $40 \mathrm{~Hz}$ uncertainty $(\approx 20 \mathrm{ppb})$ ) to extract a value of $\mu_{\mu} / \mu_{p}$ (to $\approx 120 \mathrm{ppb}$ ) for positive muons.

At the strong field of $1.7 \mathrm{~T}$ used in the experiment, $x \simeq 10.7 \gg 1$, these transitions correspond essentially to pure muon spin flip. Lorentz violating energy shifts, $\delta \nu_{12}$ and $\delta \nu_{34}$, to the transition frequencies, $\nu_{12}$ and $\nu_{34}$, in muonium coming from the standard model extension, may then be attributed to the muon parameters alone of the theory. The prediction, described in detail in 9 is :

$$
\delta \nu_{12} \approx-\delta \nu_{34} \approx \tilde{b}_{3}^{\mu} / \pi
$$

where $\tilde{b}_{3}^{\mu} \equiv b_{3}^{\mu}+d_{30}^{\mu} m_{\mu}+H_{12}^{\mu}$ are laboratory frame parameters. Precision microwave spectroscopy on muonium can measure or set limits on these symmetry violating terms, with sensitivity at the Planck scale levell.

Predicting $\nu_{12}$ and $\nu_{34}$ from QED requires knowledge of many atomic constants; $m_{\mu}, \mu_{\mu}$, and $\Delta \nu$ in particular, as well as the calculation of higher order QED radiative corrections, and small electroweak and hadronic radiative corrections. The relevant constants (and hence calculation results) are not known to as high accuracy as the experimental determinations of $\nu_{12}$ and $\nu_{34}$. Comparing predictions for $\nu_{12}$ and $\nu_{34}$ (based on independent determinations of the required atomic constants) with the experimental results has poor sensitivity to the non-standard model energy shifts $\delta \nu_{12}$ and $\delta \nu_{34}$.

The most powerful signature of $\mathrm{CPT}$ and Lorentz violation in this case comes from the observation that since the laboratory rotates with the earth, and the parameters of CPT and Lorentz violation involve spatial components in a fixed celestial frame, the experimentally observed $\nu_{12}$ and $\nu_{34}$ may oscillate about a mean value at the earth's sidereal frequency $\Omega=2 \pi / 23 \mathrm{hr} 56 \mathrm{~min}$ with amplitudes $\delta \nu_{12}$ and $\delta \nu_{34}$. An experimental limit on the oscillation amplitude $\delta \nu_{12}$ implies constraints on the celestial frame parameters :

$$
\frac{1}{\pi}|\sin \chi| \sqrt{\left(\tilde{b}_{X}^{\mu}\right)^{2}+\left(\tilde{b}_{Y}^{\mu}\right)^{2}} \leq \delta \nu_{12}
$$

in which $\chi \sim 90^{\circ}$ is the angle between the earth's rotational axis, $\hat{Z}$, and the quantization axis defined by the laboratory magnetic field at Los Alamos (parameters and coordinates are described in 9 ). 
The experimental signature has several nice features. The sum of the transition frequencies, $\nu_{12}+\nu_{34}$, is equal to the ground state hyperfine splitting, $\Delta \nu$, and since we expect (see Eqn. 3) $\delta \nu_{12}+\delta \nu_{34} \approx 0$, no sidereal variation is expected in the hyperfine interval. However, the difference in transition frequencies $\delta \nu_{12}-\delta \nu_{34} \approx 2 \tilde{b}_{3}^{\mu} / \pi$ would exhibit the maximum sidereal variation. The expectation that sidereal variations in $\nu_{12}$ and $\nu_{34}$ would be exactly out of phase allows the above consistency check against spurious signals. We also note that at strong fields, $\nu_{34}-\nu_{12}$ is almost proportional to the magnetic moment of the muon, so we are essentially probing for a sidereal variation in the magnetic moment (while the $g$ factor stays constant to first order in the theory).

\section{Details of the Experiment and Analysis}

The measurements of $\nu_{12}$ and $\nu_{34}$ were done in a microwave magnetic resonance experiment 4 . Muonium was formed by electron capture by polarized muons (negative helicity) stopping in a krypton gas target. The muons would subsequently decay weakly via $\mu^{+} \rightarrow e^{+} \nu_{e} \bar{\nu}_{\mu}$ where the momentum and angle of the $e^{+}$is a function of the muon polarization. Since high momenta decay positrons are emitted preferentially in the muon spin direction, by driving the muon spin flip transitions $\nu_{12}$ and $\nu_{34}$ with a microwave magnetic field perpendicular to the strong static field, the angular distribution of high momenta $e^{+}$ could be changed from predominantly upstream to downstream with respect to the beam direction if the microwave field was near the resonance frequency for the transition for the given value of the static field. The apparatus is shown in Fig. 2. Resonance lines were observed by varying the magnetic field with fixed microwave frequency and by varying the microwave frequency with fixed magnetic field. A line narrowing technique 10 was used involving observation of a transition signal only from $M$ atoms which have lived considerably longer than $\tau_{\mu} \sim 2.2 \mu$ s (Fig. 3).

To search for a time dependence of $\nu_{12}$ and $\nu_{34}$, data from each resonance line run (each lasting about half an hour) were fit at the measured magnetic field strength and $\mathrm{Kr}$ pressure to determine provisional line centers for $\nu_{12}$ and $\nu_{34}$. These line centers were transformed to their values in a magnetic field strength corresponding to a free proton precession frequency of 72.320 $000 \mathrm{MHz}$. The data were taken at Kr pressures of 0.8 and $1.5 \mathrm{~atm}$, so the line centers were corrected for a small quadratic pressure shift, and were extrapolated linearly to their values at zero pressure, using a pressure shift coefficient determined from the data.

The overall results were $\nu_{12}(\exp )=1897539800$ (35) Hz (18 ppb) 


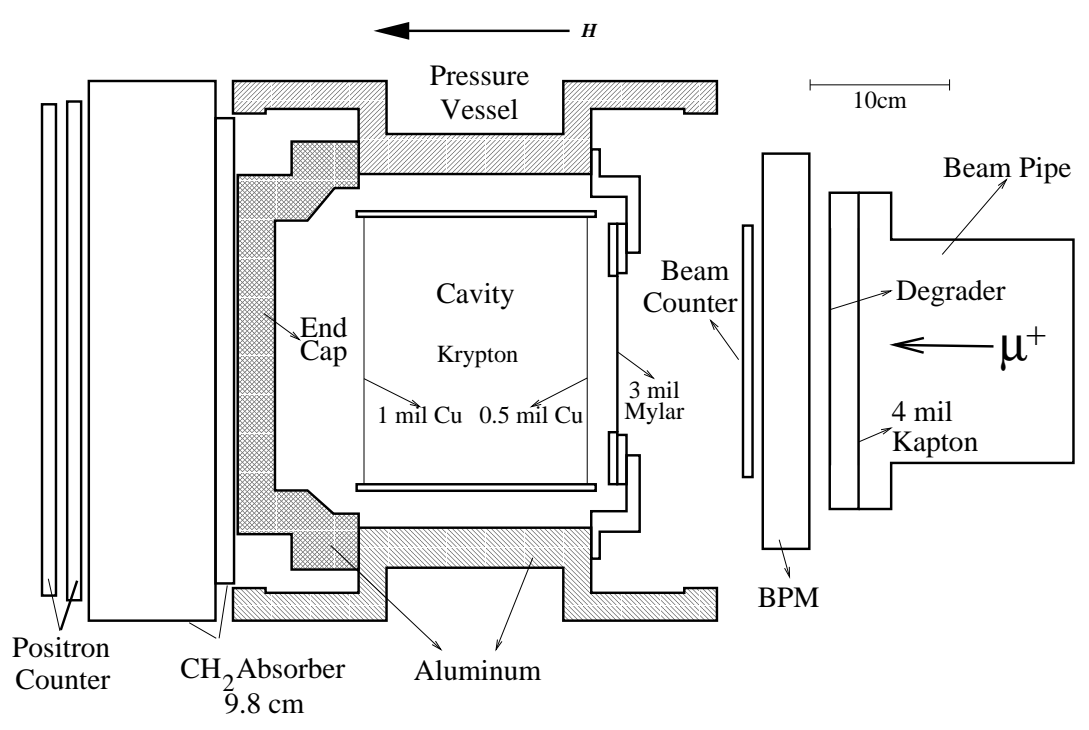

Figure 2: Schematic of the experimental apparatus.

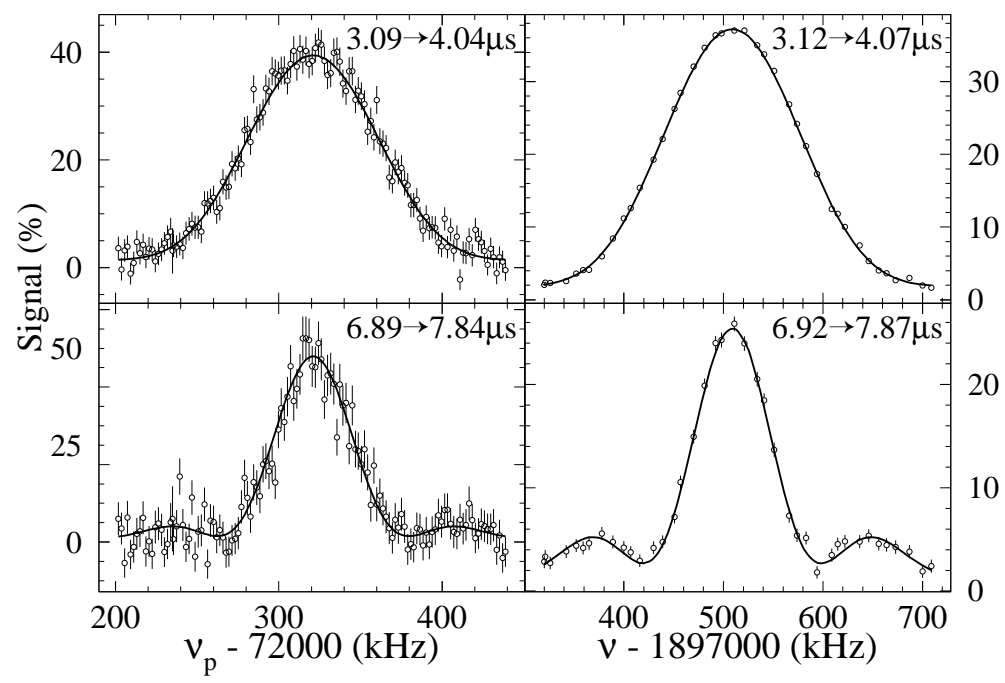

Figure 3: Muonium resonance lines (data and fit) for $\nu_{12}$ taken using magnetic field sweep on the left, and microwave frequency sweep on the right, for muonium atoms which have decayed in selected time intervals after formation. 


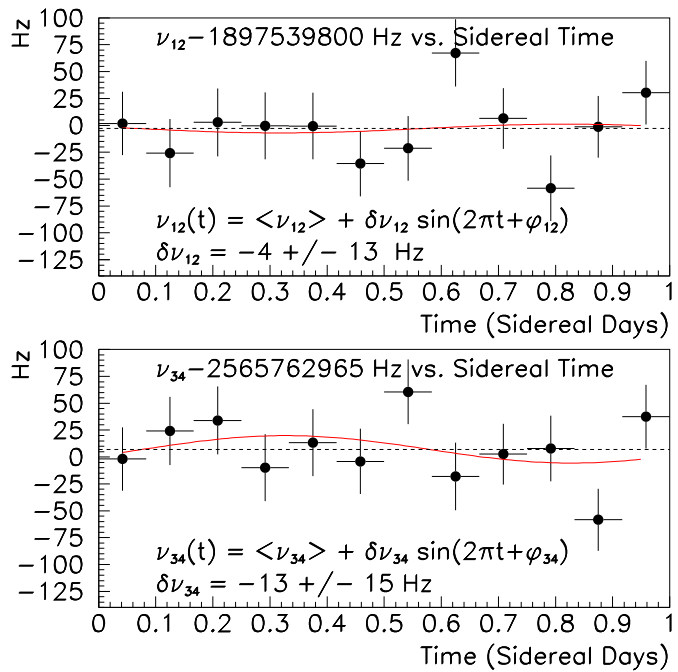

Figure 4: Two years of data on $\nu_{12}$ and $\nu_{34}$ are shown binned versus sidereal time and fit for a possible sinusoidal variation. The amplitudes are consistent with zero.

and $\nu_{34}(\exp )=2565762965(43) \mathrm{Hz}(17 \mathrm{ppb})$ where the uncertainties reflect statistical and systematic uncertainties combined. Some systematic uncertainties which were pertinent for the extraction of these final vacuum values of $\nu_{12}$ and $\nu_{34}$ for tests of QED and for extracting constants, such as uncertainties in the absolute calibration of the pressure meters or pressure shift coefficients, do not affect the extraction of possible sidereal variations at first order. The results for $\nu_{12}$ and $\nu_{34}$ were then grouped as a function of sidereal time, where time zero has been set as the time in 1995 when we obtained our first data. The data obtained in 1995 and 1996 are plotted as a function of time measured as a function of a sidereal day in Fig. A, where twelve points at $\approx 2 \mathrm{hr}$. intervals are plotted, and the vertical scale is in $\mathrm{Hz}$. The data for $\nu_{12}$ and $\nu_{34}$ were fit separately by the functions

$$
\nu_{i j}(t)=\left\langle\nu_{i j}\right\rangle+\delta \nu_{i j} \sin \left(2 \pi t+\phi_{i j}\right)
$$

where $t$ is the time in sidereal days, and the fit parameters are $\left\langle\nu_{i j}\right\rangle$, the amplitude of the possible time variation $\delta \nu_{i j}$, and the phase $\phi_{i j}$ (where no phase relation was assumed between $\nu_{12}$ and $\nu_{34}$ ). 


\section{Potential Systematic Effects}

Non-zero values for $\delta \nu_{12}$ and $\delta \nu_{34}$ could have arisen from systematic effects which lead to variations in the parameters determining the line centers - especially parameters varying with a period of $\approx 24 \mathrm{hr}$. Principal concerns are possible day-night variations of the magnetic field strength, and of the density and temperature of the $\mathrm{Kr}$ gas stopping target.

Diurnal variations of several ${ }^{\circ} \mathrm{C}$ in the experimental hall lead to oscillations in the magnetic field strength of the persistent-mode superconducting solenoid of $\leq 1 \mathrm{ppm}$. Changes of $0.05 \mathrm{ppm}$ in the field strength were easily resolved, and the oscillation's effects on the line centers were accounted for in extracting the line centers. Temperature changes also affected the diamagnetic shielding constant of the water in the NMR probes used to monitor the field (the probes were not temperature-stabilized, but were in good thermal contact with the microwave cavity which was temperature-stabilized to $0.1{ }^{\circ} \mathrm{C}$ ). A conservative upper limit of $2{ }^{\circ} \mathrm{C}$ diurnal variation in water temperature (estimated from a simple thermal transport model) would change the NMR frequencies by 0.02 ppm, leading to errors in the line centers of about $2.5 \mathrm{~Hz}$; of opposite sign for $\nu_{12}$ and $\nu_{34}$. This potential effect is well below the statistical sensitivity for sidereal variations of 12 to $15 \mathrm{~Hz}$.

The effect of the diurnal variation of Kr pressure has been evaluated. The $76 \mu \mathrm{m}$ mylar front end window to the $\mathrm{Kr}$ stopping target flexed with daynight variations of the external atmospheric pressure. This induced fractional oscillations in the $\mathrm{Kr}$ gas target pressure which were measured to be about $2.5 \times 10^{-4}$. Through pressure shift coefficients of about $-16.5 \mathrm{kHz} / \mathrm{atm}$ for $\nu_{12}$ and $-19.5 \mathrm{kHz} / \mathrm{atm}$ for $\nu_{34}$, the resulting shifts in the line centers (typically $7.5 \mathrm{~Hz}$ in $\nu_{34}$ and $6 \mathrm{~Hz}$ in $\nu_{12}$ ) were automatically accounted for in performing the extrapolation to zero pressure, and do not contribute any significant time variation to $\nu_{i j}$.

The pressure shift coefficients depend on the average velocities of the atoms, and so are functions of temperature. The fractional changes in the transition frequencies with temperature (measured in hydrogen and its isotopes 11 ) are roughly $1 \times 10^{-11}{ }^{\circ} \mathrm{C}^{-1} \mathrm{Torr}^{-1}$. Given the temperature stability of the $\mathrm{Kr}$ gas of about $0.1{ }^{\circ} \mathrm{C}$, temperature dependent errors introduced into the extrapolation of the line centers to their vacuum values would be limited to a few $\mathrm{Hz}$, below the statistical sensitivity of our test.

Other potential concerns involve the two frequency references used in the experiment - the proton precession frequency forming the basis of the magnetic field determination, and the Loran-C $10 \mathrm{MHz}$ frequency reference used for the NMR and microwave frequency synthesizers. The Loran-C standard is 
based on hyperfine transitions in Cs with $m_{F}=0$, and so is insensitive to any preferred spatial orientation, and would not introduce a signature for Lorentz violation into the spectroscopic measurements. Significant oscillations in the Loran-C are precluded by the null results in both $\nu_{12}+\nu_{34}$ and $\nu_{34}-\nu_{12}$. Finally, bounds on clock comparisons of ${ }^{199} \mathrm{Hg}$ and ${ }^{133} \mathrm{Cs} 1213$ place limits on the Lorentz violating energy shifts in the precession frequency of a proton of $10^{-27} \mathrm{GeV}$, which imply the NMR measurements are free of shifts well below the $\mathrm{Hz}$ level.

\section{Results}

The amplitudes for $\delta \nu_{12}$ and $\delta \nu_{34}$ independently are consistent with zero, $-4 \pm$ $13 \mathrm{~Hz}$ and $-13 \pm 15 \mathrm{~Hz}$ respectively. However, the standard model extension predicts the phase relation $\delta \nu_{12} \approx-\delta \nu_{34}$. Plots of $\nu_{12} \pm \nu_{34}$ versus sidereal time are shown in Figure 5 , and fit for a sinusoidal variation (as in Eqn.5) where a common phase is assumed between $\nu_{12}$ and $\nu_{34}$. No sinusoidal variation with sidereal time within $\pm 20 \mathrm{~Hz}$ is found in either the sum or difference, which yields a $68 \%$ confidence level (one sigma) limit on the non-

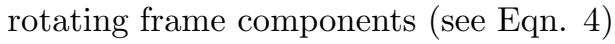

$$
\sqrt{\left(\tilde{b}_{X}^{\mu}\right)^{2}+\left(\tilde{b}_{Y}^{\mu}\right)^{2}} \leq 2 \times 10^{-23} \mathrm{GeV} .
$$

The figure of merit of these results as a test of CPT violation is taken as :

$$
2 \sqrt{\left(\tilde{b}_{X}^{\mu}\right)^{2}+\left(\tilde{b}_{Y}^{\mu}\right)^{2}} / m_{\mu} \leq \frac{2 \pi\left|\delta \nu_{12}\right|}{m_{\mu}} \approx 5 \times 10^{-22} .
$$

which is comparable to the dimensionless scaling factor $m_{\mu} / M_{P} \sim 10^{-20}$. The limits on $\delta \nu_{34}$ and $\delta\left(\nu_{34}-\nu_{12}\right)$ yield similar values.

\section{Conclusions}

No unambiguous violation of CPT or Lorentz invariance has been observed in this system, or any other, despite many sensitive tests performed over the more than 40 years since the first experiments of Hughes and Dreve 14. The null results presented here are the first test for sidereal variations in the interactions of the muon arising from CPT and Lorentz violation, and set the first limits on the associated combination of muon parameters of the standard model extension at the level of $2 \times 10^{-23} \mathrm{GeV}$, representing Planck scale sensitivity, and almost an order of magnitude improvement in sensitivity over previous results in the muon sector $\mathrm{B}$. Further improvement in the muon parameters may 

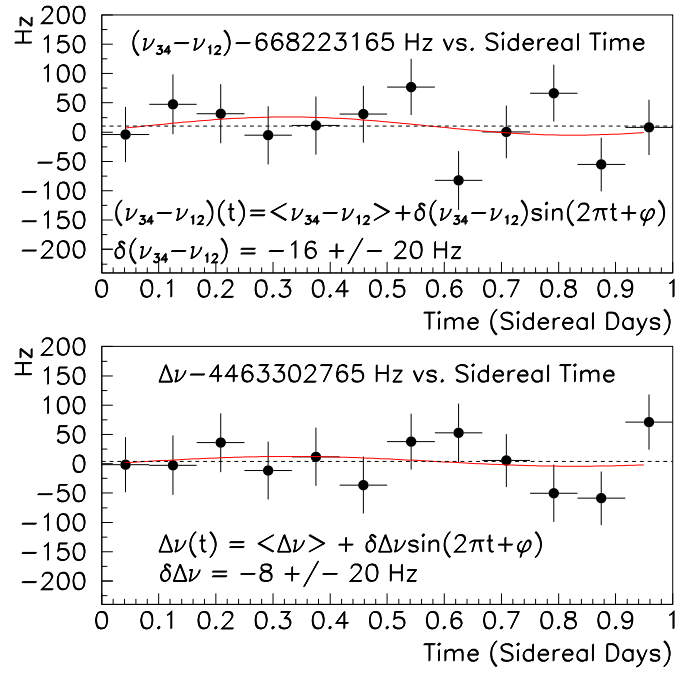

Figure 5: Two years of data on $\nu_{12}-\nu_{34}$ and $\nu_{12}+\nu_{34}=\Delta \nu$ are shown binned versus sidereal time and fit for a possible sinusoidal variation. The amplitudes are consistent with zero.

come from the ongoing muon g-2 experiment (see M. Deile's contribution in this volume). Improvement from a new muonium experiment measuring ground state hyperfine transitions will likely require a more intense muon source, as the current experiment was statistics limited.

\section{Acknowledgments}

Our thanks go to V.A. Kostelecký for helpful discussions and for organizing such an interesting meeting. Thanks also to the U.S. DOE and BMBF (Germany) for supporting this research.

\section{References}

1. W. Liu et al., Phys. Rev. Lett. 82, 711 (1999) and references therein.

2. V. Meyer et al., Phys. Rev. Lett. 84, 1136 (2000).

3. P.J. Mohr and B.N. Taylor, Rev. Mod. Phys. 72, 351 (2000).

4. L. Willmann et al., Phys. Rev. Lett. 82, 49 (1999).

5. V.A. Kostelecký and R. Potting, Nucl. Phys. B 359, 545 (1991); Phys. Lett. B 381, 89 (1996). 
6. D. Colladay and V.A. Kostelecký, Phys. Rev. D 55, 6760 (1997); Phys. Rev. D 58, 116002 (1998).

7. V.A. Kostelecký, ed., CPT and Lorentz Symmetry (World Scientific, Singapore, 1999).

8. V.W. Hughes et al., Phys. Rev. Lett. 87, 111804 (2001).

9. R. Bluhm, V.A. Kostelecký, and C.D. Lane, Phys. Rev. Lett. 84, 1098 (2000).

10. M.G. Boshier et al., Phys. Rev. A 52, 1948 (1995).

11. C.L. Morgan and E.S. Ensberg, Phys. Rev. A 7, 1494 (1973).

12. V.A. Kostelecký and C.D. Lane, Phys. Rev. D 60, 116010 (1999).

13. J.D. Prestage et al., Phys. Rev. Lett. 54, 2387 (1985), C.J. Berglund et al., Phys. Rev. Lett. 75, 1879 (1995) and references therein.

14. V.W. Hughes, H.G. Robinson and V. Beltran-Lopez, Phys. Rev. Lett. 4, N504 (1960), R.W.P. Drever, Philos. Mag. 6, 683 (1961). 